\title{
Mathematical modelling and optimization of biomass-plastic fixed-bed downdraft co- gasification process
}

\author{
Igor Donskoy, ${ }^{1, *}$ \\ ${ }^{1}$ Kutateladze Institute of Thermophysics of SB RAS, 630090, Novosibirsk, Russia Lavrentieva st., 1
}

\begin{abstract}
Co-gasification of woody biomass and polyethylene is studied using mathematical modeling. The gasification process is downdraft fixedbed. Comparison of modeling results with some experimental data is made. Influence of biomass/plastic ratio and air equivalence ratio on gasification efficiency is investigated.
\end{abstract}

\section{Introduction}

Low-grade fuels, such as forest and agricultural or municipal wastes, often cannot be converted into energy in efficient way. This is concerned with high content of noncombustible matter and possible hazardous contaminants formation (PAH, chlorine compounds, etc.). In this way, co-conversion of low-grade fuels with traditional fuels is under interest $[1,2]$.

Plastic utilization is an urgent problem because polymeric matter forms the main part of municipal wastes (both in mass fraction and in heat value). But thermal behaviour of plastic often leads to conversion process destabilization due to particles agglomeration $[3,4]$.

Co-gasification of woody biomass and plastic in downdraft fixed-bed reactor is studied at the present paper using mathematical modeling. Attainability of computed regimes is discussed.

\section{Input data for modelling}

Mathematical model of the gasification process was developed and used earlier in works $[5,6]$. Geometric parameters were taken from [7]. Fuel consumption is $30 \mathrm{~kg} / \mathrm{h}$. Active bed height is $2 \mathrm{~m}$, reactor diameter is $0.44 \mathrm{~m}$. Air flow is varied from 30 to $60 \mathrm{Nm} 3 / \mathrm{h}$. Plastic mass fraction in fuel mixture changes from $0 \%$ to $50 \%$. Mean particle size is $5 \mathrm{~cm}$ for biomass and $5 \mathrm{~mm}$ for plastic. Properties of wood and plastic are listed at table 1. During pyrolysis tar formation occurs. Simplifications are made as following. Tar has uniform composition $\left(\mathrm{CH}_{0.6}\right)$, and tar fraction in pyrolysis products is constant for each fuel. Ethylene in pyrolysis gas is formed only from plastic and its fraction in pyrolysis products also is constant (30\% on organic matter basis). Two compositions of wood were used: the

\footnotetext{
*Corresponding author: donskoy.chem@mail.ru
} 
first was taken from paper [7] to compare modeling results with experiment (wood-1); the second is averaged wood composition based on our previous researches [8] (wood-2).

Some heterogeneous reactions are taken into account in computations. Kinetic parameters of these reactions are listed at table $2[8,9]$. Decomposition of tar has the same kinetic parameters for both fuels. (CGE).

Key process characteristics are gas composition, temperature and cold gas efficiency

Table 1. Composition of wood and plastic.

\begin{tabular}{|c|c|c|c|}
\hline Fuel property & Wood-1 & Wood-2 & Plastic \\
\hline $\mathrm{A}^{\mathrm{d}}, \%$ & 29 & 20.0 & 0.1 \\
\hline $\mathrm{W}^{\mathrm{r}}, \%$ & 0.45 & 0.45 & 0.0 \\
\hline $\mathrm{V}^{\text {daf }} \%$ & 85.9 & 84.65 & 99.9 \\
\hline $\mathrm{C}^{\text {daf }} \%$ & 35.3 & 41.04 & 85.0 \\
\hline $\mathrm{H}^{\text {daf }} \%$ & 7.60 & 7.01 & 14.0 \\
\hline $\mathrm{O}^{\text {daf }} \%$ & 57.2 & 51.93 & 0.0 \\
\hline Tar fraction in pyrolysis products & 0.2 & 0.2 & 0.9 \\
\hline
\end{tabular}

Table 2. Kinetic parameters for heterogeneous reactions of biomass and plastic.

\begin{tabular}{|c|c|c|c|c|}
\hline \multirow{2}{*}{ Reaction } & \multicolumn{2}{|c|}{ Biomass } & \multicolumn{2}{c|}{ Plastic } \\
\cline { 2 - 5 } & $\mathrm{k}_{0}$ & $\mathrm{E}_{\mathrm{a}}, \mathrm{kJ} / \mathrm{mol}$ & $\mathrm{k}_{0}$ & $\mathrm{E}_{\mathrm{a}}, \mathrm{kJ} / \mathrm{mol}$ \\
\hline Pyrolysis & $5.4 \cdot 10^{3}, 1 / \mathrm{s}$ & 96 & $1.7 \cdot 10^{14}, 1 / \mathrm{s}$ & 220 \\
\hline $\mathrm{C}+\mathrm{O}_{2}$ & $2.4 \cdot 10^{8}, \mathrm{~m} / \mathrm{s}$ & 142 & - & - \\
\hline $\mathrm{C}+\mathrm{CO}_{2}$ & $1.32 \cdot 10^{9}, \mathrm{~m} / \mathrm{s}$ & 259 & - & - \\
\hline $\mathrm{C}+\mathrm{H}_{2} \mathrm{O}$ & $9.3 \cdot 10^{7}, \mathrm{~m} / \mathrm{s}$ & 175 & - & 150 \\
\hline $\begin{array}{c}\text { Tar } \\
\text { decomposition }\end{array}$ & $5 \cdot 10^{8}, 1 / \mathrm{s}$ & 150 & $5 \cdot 10^{8}, 1 / \mathrm{s}$ & \\
\hline
\end{tabular}

\section{Comparison with reference data}

Computations were made using mathematical model and set of kinetic parameters listed above. Input data for stationary regimes from [7] was used. Wood composition used is wood-1 at table 1. Comparison results are shown at table 3 . One can see that the model could predict accurately a few number of process characteristics such as output syngas volume rate and concentration of several species (for example, $\mathrm{CO}_{2}$ ). Other characteristics could be calculated with qualitative agreement only. Gasification regimes of biomass without plastic are reproduced better. The difference between measured and calculated values of temperature is most likely concerned with underestimation of heat losses.

To make modeling results more close to experiment it is necessary to improve pyrolysis stage modeling block. This process is considered as one-step overall-reaction. The most of modeling error is a result of incorrect estimate of hydrocarbons concentration.

\section{Results of optimization study}

Computations were also made to estimate optimal co-gasification conditions. Wood-2 was used as a wood composition here. Variable parameters are air equivalence ratio and plastic mass fraction in fuel mixture. The results are presented at fig. 1. 
Table 3. Comparison of modelling results and experimental data (Garcia-Bacaioca et al., 2008).

\begin{tabular}{|c|c|c|c|c|c|c|c|c|}
\hline No. & P1-1 & P1-2 & P1-3 & P2-1 & P2-2 & P2-3 & P3-1 & P3-2 \\
\hline $\mathrm{G}_{\text {air }}, \mathrm{Nm}^{3} / \mathrm{h}$ & 43 & 50 & 60 & 38.7 & 51.9 & 59.3 & 40 & 52.5 \\
\hline $\mathrm{G}_{\text {fuel }}, \mathrm{kg} / \mathrm{h}$ & 34.4 & 40.4 & 46 & 29.6 & 35.3 & 37.9 & 33.4 & 30.9 \\
\hline Plastic fraction, $\%$ & 0 & 0 & 0 & 9.6 & 9.8 & 9.5 & 15.2 & 17.4 \\
\hline $\mathrm{t}_{\mathrm{air}},{ }^{\circ} \mathrm{C}$ & 75 & 70 & 113 & 85 & 116 & 139 & 109 & 126 \\
\hline \multirow{2}{*}{$\mathrm{G}_{\mathrm{gas}}, \mathrm{Nm}^{3} / \mathrm{h}$} & 56.8 & 66.9 & 82.4 & 59.5 & 80.3 & 94.3 & 61.9 & 81.5 \\
\hline & 63.6 & 74.6 & 85.9 & 62.4 & 76.7 & 83.3 & 72.9 & 75.6 \\
\hline \multirow{2}{*}{$\mathrm{N}_{2}, \%$} & 59.3 & 57.4 & 56.0 & 50.9 & 50.5 & 49.2 & 50.4 & 50.8 \\
\hline & 52.8 & 52.4 & 54.6 & 48.5 & 52.9 & 55.6 & 42.8 & 54.3 \\
\hline \multirow{2}{*}{$\mathrm{O}_{2}, \%$} & 0.5 & 0.7 & 0.4 & 0.3 & 0.5 & 0.4 & 0.1 & 0.6 \\
\hline & 0.0 & 0.0 & 0.0 & 0.0 & 0.0 & 0.0 & 0.0 & 0.0 \\
\hline \multirow{2}{*}{$\mathrm{CO}_{2}, \%$} & 17.3 & 14.7 & 17.2 & 12.0 & 10.6 & 10.8 & 9.6 & 7.3 \\
\hline & 16.4 & 16.6 & 16.2 & 14.2 & 13.8 & 13.6 & 14.9 & 12.2 \\
\hline \multirow{2}{*}{$\mathrm{CO}, \%$} & 10.1 & 10.2 & 9.4 & 19.2 & 20.1 & 20.2 & 19.6 & 22.5 \\
\hline & 11.9 & 11.8 & 11.9 & 13.3 & 13.0 & 12.8 & 12.4 & 13.5 \\
\hline \multirow{2}{*}{$\mathrm{H}_{2}, \%$} & 11.2 & 15.1 & 14.8 & 13.9 & 13.7 & 15.1 & 14.4 & 14.6 \\
\hline & 18.3 & 18.7 & 16.7 & 22.3 & 18.6 & 16.3 & 26.8 & 17.7 \\
\hline \multirow{2}{*}{$\mathrm{CH}_{4}, \%$} & 0.8 & 0.9 & 1.2 & 2.0 & 2.5 & 2.4 & 3.1 & 3.0 \\
\hline & 0.0 & 0.0 & 0.0 & 0.0 & 0.0 & 0.0 & 0.6 & 0.0 \\
\hline \multirow{2}{*}{$\mathrm{C}_{2}, \%$} & 0.7 & 0.9 & 1.1 & 0.8 & 1.2 & 0.9 & 1.7 & 1.6 \\
\hline & 0.0 & 0.0 & 0.0 & 1.1 & 1.1 & 1.0 & 1.6 & 1.7 \\
\hline \multirow{2}{*}{$\mathrm{Q}_{\mathrm{gas}}, \mathrm{MJ} / \mathrm{Nm}^{3}$} & 3.2 & 3.7 & 3.8 & 5.6 & 6.1 & 6.1 & 6.7 & 6.9 \\
\hline & 3.5 & 3.5 & 3.3 & 4.7 & 4.3 & 4.0 & 5.6 & 4.6 \\
\hline \multirow{2}{*}{ CGE, \% } & 44.5 & 48.5 & 52.1 & 66.3 & 81.9 & 90.2 & 65.9 & 93.6 \\
\hline & 50.8 & 51.2 & 48.9 & 63.6 & 59.2 & 56.1 & 70.8 & 62.3 \\
\hline \multirow{2}{*}{$\mathrm{t}_{\text {out }},{ }^{\circ} \mathrm{C}$} & 787 & 687 & 687 & 620 & 806 & 957 & 779 & 811 \\
\hline & 888 & 875 & 939 & 797 & 888 & 962 & 641 & 912 \\
\hline
\end{tabular}
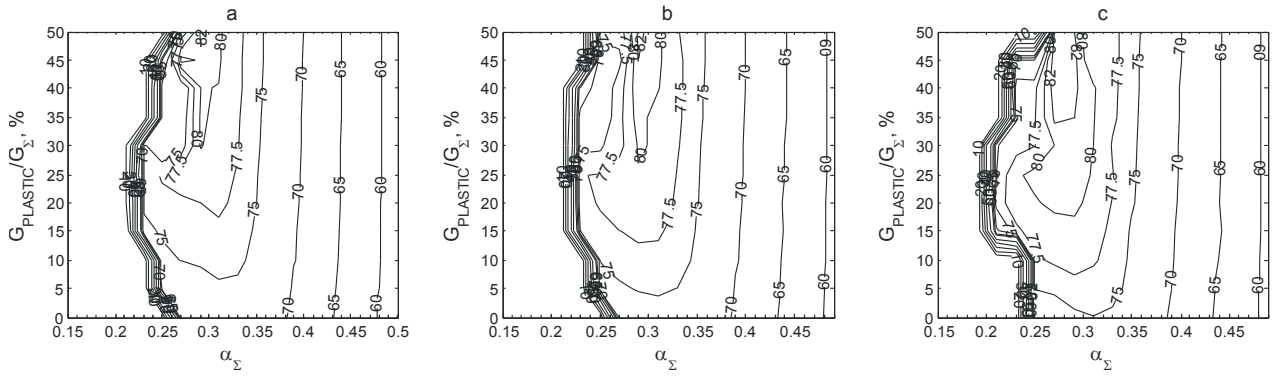

Fig. 1. Cold gas efficiency (\%) dependence on summary air equivalence ratio $(\alpha \Sigma)$ and mass fraction of plastic (Gplastic/Gs): a - initial air temperature is $30^{\circ} \mathrm{C} ; \mathrm{b}-100^{\circ} \mathrm{C} ; \mathrm{c}-200^{\circ} \mathrm{C}$.

One can see that cold gas efficiency has well-known dependence on air equivalence ratio [10]. It should be noted that line of maximum CGE for mixtures of fuels with highvolatiles content $(>80 \%)$ is very close to a boundary of thermally stable gasification conditions. The more is initial air temperature the wider are boundaries of efficient gasification regimes. Plastic addition increases CGE too. But some regimes at fig. 1 cannot be attained, because the more plastic fraction the more feasibility of the bed agglomeration. So the co-gasification efficiency limit would be estimated more precisely using correlations 
of the critical plastic fraction (e.g. fraction that provide bed structure stability) with process conditions. This task is a possible topic of future works.

This work was carried out at the Kutateladze Institute of Thermophysics SB RAS and financially supported by the Russian Science Foundation (project number 15-19-10025).

\section{References}

1. U. Arena, Waste Manag., 32, 625-639 (2012).

2. D.R. McIlveen-Wright, F. Rinto, L. Armesto et al., Fuel Proc. Tech. 87, 793-801 (2006).

3. M.L. Mastellone, U. Arena, Polymer Degradation and Stability 85, 1051-1058 (2004).

4. S.M. Lera, Tesis Doctoral (PhD) (Univ. de Zaragoza, 2015)

5. I.G. Donskoi, A.V. Keiko, A.N. Kozlov et al., Thermal Eng. 60, 904-909 (2013).

6. I.G. Donskoy, MATEC Web of Conf. 72, 01026 (2016).

7. P. Garcia-Bacaioca, J.F. Mastral, J. Ceamanos et al., Biores. Tech. 99, 5485-5491 (2008).

8. A. Kozlov, D. Svishchev, I. Donskoy, A.V. Keiko, JTAC 109, 1311-1317 (2012).

9. H. Bockhorn, A. Hornung, U. Hornung, D. Schawaller, JTAC 48, 93-109 (1999).

10. M.J. Prins, Proefschrift (Tech. Univ. Eindhoven, 2005). 